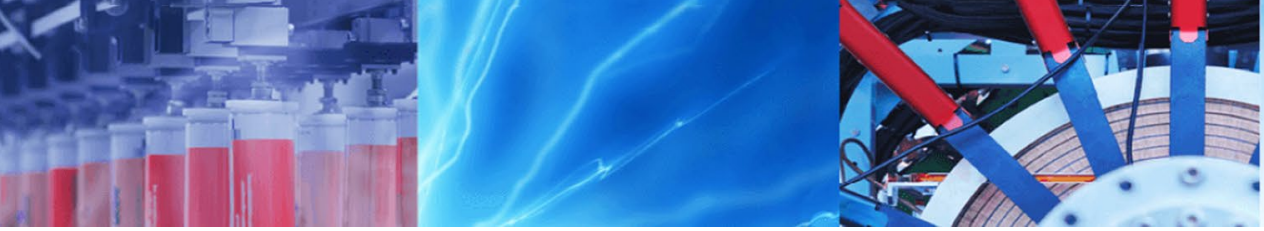

Research Article

\title{
Experimental study on energy consumption in rotating machinery caused by misalignment
}

\author{
Bouchra Abouelanouar ${ }^{1}\left[\right.$ [D $\cdot$ Ali Elkihel ${ }^{2} \cdot$ Hassan Gziri $^{2}$
}

Received: 22 October 2019 / Accepted: 10 June 2020 / Published online: 15 June 2020

(c) Springer Nature Switzerland AG 2020

\begin{abstract}
In this study we present an evaluation of the energy consumption in the case of shaft misalignment. Misalignment is one of the most common defects in rotating machinery that causes noticeable losses of energy. The proposed methodology focuses on finding a correlation between vibration level and energy consumption for different degrees of misalignment. In fact, the monitoring of energy consumption became a necessity for industries for the implementation of the appropriate maintenance strategy. In order to achieve this, vibration analysis is a measurement tool used to detect and diagnose rotating machinery faults, such as misalignment, unbalance and bearing defects. The results of this experimental investigation show that the rate of energy consumption depends highly on the degree of shaft misalignment, especially, in the case of parallel misalignment. Also, vibration analysis has been proven to be an effective tool to early detect misalignment shaft and monitoring electrical energy consumption.
\end{abstract}

Keywords Predictive maintenance · Energy consumption · Rotating machinery $\cdot$ Misalignment $\cdot$ Vibration analysis

\section{Introduction}

The fight against climate change, $\mathrm{CO}_{2}$ emissions and the extra cost of the energy bills are pushing industries to reduce their energy consumption. The gains may be relieved in the building, the utilities (heating, air, lighting...) and the process. Currently, the management of energy consumption in production processes, which account for about $2 / 3$ of total consumption, has become a necessity for companies to ensure their competitiveness. In this context, the realization of energy audits is now a priority because they allow a methodological analysis of energy use and consumption. This audit is considered as a first step to reduce energy consumption by providing a detailed inventory of the energy performance of the company and its installations. Finally, solutions for improving energy efficiency are proposed based on the results of the energy audit. [1].

For this purpose, predictive maintenance is a powerful tool to optimize the availability of manufacturing and production plants, cost efficiency of maintenance work, and can also be used in the evaluation of energy consumption in the case of damage. Predictive maintenance provides factual data on the actual mechanical condition of each machine and the operating efficiency of each process $[2$, 3]. The nondestructive techniques usually used for predictive maintenance program are vibration monitoring, infrared thermography, ultrasound testing and oil analysis. Each technique has a unique data and its sensitivity depends on the severity of the defect [4]. Vibration analysis has always been known as the most widely used and powerful method for rotating machine monitoring.

$\triangle$ Bouchra Abouelanouar, b.abouelanouar@ump.ac.ma; Ali Elkihel, alielkihel@yahoo.fr; Hassan Gziri, hgziri@gmail.com | ${ }^{1}$ Laboratoire de Génie Industriel et Génie Sismique, Ecole Nationale des Sciences Appliquées, Université Mohamed Premier, Oujda, Morocco. ${ }^{2}$ Laboratoire Ingénierie, Management Industriel et Innovation, Faculté des Sciences et Techniques de Settat, Université Hassan 1er, Casablanca, Morocco. 
After reading some literatures, it was found that misalignment and unbalancing are the most common cause $(\sim 70 \%)$ of machine vibration that are causing energy loss and reduce the life of rotating machines [5]. Statistically speaking, industries are losing billions of dollars a year because of misaligned machines [6]. Furthermore, misaligned machine is more prone to failure due to increased loads on bearing and couplings; that would lead to increased energy consumption [7]. Several researches were carried out in the experimental and analytical study of misalignment considering the changes in vibration response and its impact on the consumption of electrical energy [8-12]. For example, according to H.A. Gaberson [13], nearly $3 \%$ of energy losses are due to misalignment, while the unbalance leads to losses of less than $1 \%$. E. Estupinan et al. [14], presented an analysis of energetic losses generated by misalignment, which was based on vibration measurements. It was proved economically that the implementation of predictive maintenance reduces energy consumption and keeping low levels of vibration.

In practice, vibration and temperature can provide detection of alignment issues and perform a rotating machines condition monitoring. However, by comparison of their effectiveness in early detection, vibration analysis seems better suited and sensitive for weak faults [15]. In this context, the paper presents the potential of vibration analysis technique for shaft misalignment diagnostic. The relationship between the machine vibration level and its power consumption is established. That is why we are going to focus our study on the collection of vibration data to identify energy consumption generated by both parallel and angular misalignment. To accomplish this objective, this paper is organized as follows: a theoretical approach of vibrations generated by misalignment is presented in Sect. 2. The experiment setup and data collection process are provided in Sect. 3. Vibrations and electric power measurements are illustrated in Sect. 4. In Sect. 5, we present the main results and discussion. The paper concludes with a summary recapping the main advantages of the proposed method and future work in Sect. 6.

\section{Theoretical approach}

\subsection{Misalignment}

Shaft misalignment is the phenomenon that occurs when the components that are coaxial by design are not actually coaxial [10]. Several symptoms allow the detection of misalignment, we cite by way of example: premature wear of bearings, seals, shafts and couplings, high axial and radial vibration levels, high temperatures in the bearing housings. Improper aligning of shafts can lead to coupling noise, overheating, vibrations and degradation of components such as rolling bearings. Correct machine alignment provides increased production time, less noise and vibration and therefore reduces maintenance costs.

In practice, there are three types of Shaft misalignment: parallel, angular and combined misalignment as shown in Fig. 1. It must be noticed that detecting a 'small' misalignment does not imply that the machine is defective; whereas it is a sign of excessive loads that can easily lead to premature failure. Consequently, companies impose their own acceptable tolerance for judging the severity of misalignment [16].

\subsection{Vibration generated by misalignment}

Rotating machine failures are mostly caused by undesirable static or dynamic forces. In fact, Shaft misalignment imposes additional forces on rotors, bearings, couplings and seals, which leading to mechanical failures and therefore the presence of vibrations. It is important to note that only dynamic forces can cause vibrations in machines. The understanding of the vibrations based on the calculation of the forces is extremely difficult, that's why vibration sensors are designed to measure motion, not force [6]. Thus, the vibration signature from shaft misalignment appears as peaks at running speed $(1 \times)$ or multiples of running speed $(2 x, 3 x$, etc.). Several research studies have attempted to demonstrate that the $2 \times$ and $4 \times$ components show the most significant changes, and that the $1 \times$ component did not change as the degree of misalignment increased $[17,9]$. It is also noticeable that the level or the amplitude of vibration does not give an idea about the severity of misalignment [5].

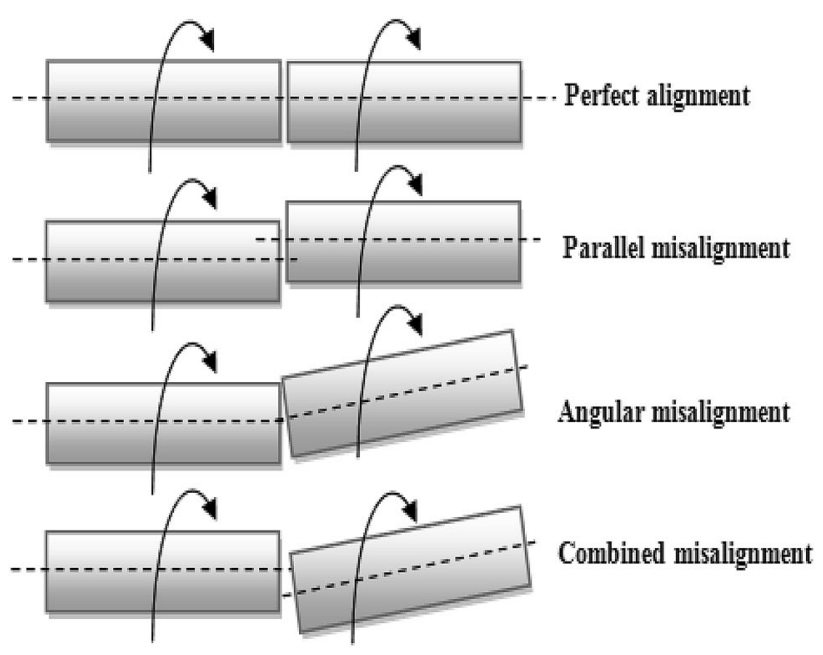

Fig. 1 Types of misalignment shaft 


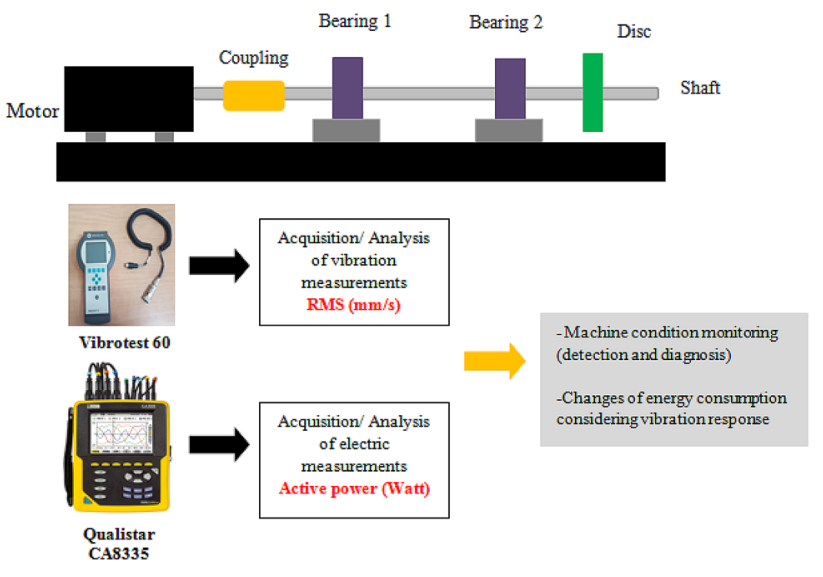

Fig. 2 Schematic diagram of experimental setup

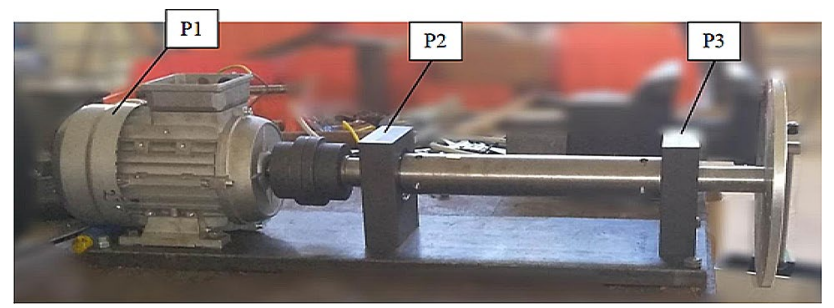

Fig. 3 Experimental set up and measurement positions. P1, P2 and P3: measurement positions in motor, bearing 1 and bearing 2, respectively

\section{Proposed methodology}

Experiments have been carried out to find out how energy consumption can be influenced by failures in a machine. In our case, we are interested in monitoring the power consumption for different misalignment conditions using vibration measurement. Figure 2 showed the schematic diagram of experimental setup and the measurement instrumentation.

Figure 3 depicts the experimental set up designed to study the shaft misalignment. It consists of an

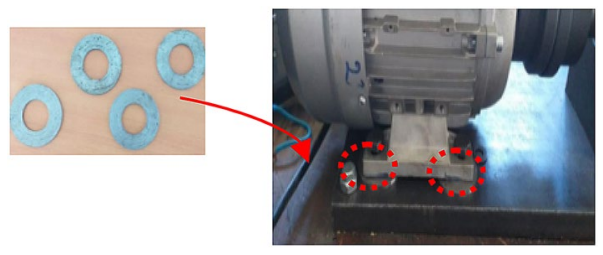

Fig. 4 Shims used to create misalignment shaft

asynchronous motor, a shaft of $20 \mathrm{~mm}$ diameter supported by two identical ball bearings and connected to the motor with a flexible coupling, and an over hung circular disc on the shaft. Three measurement points were provided on the vital components of the test bench (motor and the two bearings) to take the vibration measurement.

For comparative purposes, the two types of misalignment (parallel and angular) were created by inserting shims $(0.25 \mathrm{~mm}$ thick) under various conditions (Table 1 ) below the steel plate at corners of the motor (Fig. 4).

Table 1 describes experimental data with the conditions of parallel and angular misalignment.

This set up is used for measuring vibrations in terms of acceleration in axial and radial directions. Thus, electrical measurements have been taken in 'healthy' and defective cases to illustrate the effect of misalignment on energy consumption.

\section{Results analysis}

\subsection{Vibration measurements results}

The vibration measurements were taken in three positions: Motor (P1) and bearings of the two bearings (P2 and P3). The rotation speed of the shaft was set in 2980 rpm.

Table 1 Experimental protocol

\begin{tabular}{|c|c|c|c|c|}
\hline \multirow[t]{2}{*}{ Sensor position } & \multicolumn{3}{|c|}{ Misalignment condition } & \multirow[t]{2}{*}{ Measuring position } \\
\hline & Aligned shaft & $\begin{array}{l}\text { Parallel misalign- } \\
\text { ment }(\mathrm{mm})\end{array}$ & $\begin{array}{l}\text { Angular misalign- } \\
\text { ment }\left({ }^{\circ}\right)\end{array}$ & \\
\hline \multirow[t]{3}{*}{ Axial } & 0 & $C_{p} 1=0.25$ & $C_{A} 1=1$ & \multirow{3}{*}{$\begin{array}{l}\text { P1: Motor } \\
\text { P2: Bearing } 1 \\
\text { P2: Bearing } 2\end{array}$} \\
\hline & & $C_{p} 2=0.5$ & $C_{A} 2=2$ & \\
\hline & & $C_{p} 3=1$ & $C_{A} 3=3$ & \\
\hline \multirow[t]{3}{*}{ Radial } & 0 & $C_{p} 1$ & $C_{A} 1$ & \\
\hline & & $C_{p} 2$ & $\mathrm{C}_{\mathrm{A}} 2$ & \\
\hline & & $C_{p} 3$ & $C_{A} 3$ & \\
\hline
\end{tabular}


Table 2 RMS values in the healthy case (aligned shaft)

\begin{tabular}{lll}
\hline Measuring position & $\mathrm{RMS}(\mathrm{mm} / \mathrm{s})$ & \\
\cline { 2 - 3 } & Radial direction & Axial direction \\
\hline P1 & 0.553 & 0.498 \\
P2 & 0.664 & 0.557 \\
P3 & 0.496 & 0.337 \\
\hline
\end{tabular}

Tables 2, 3 and 4 show the results obtained from vibration level by measuring the RMS value for each misalignment conditions described in Table 1.

According to ISO 10816 (Fig. 5) prescribing the vibration severity, for small machines, the satisfactory limits of vibration are $1.8 \mathrm{~mm} / \mathrm{s}$. It can be seen from Tables 3 and 4 that the RMS values in the case of both angular and parallel misalignment exceed this limit.

\subsection{Energy balance}

Now, the electrical characteristics (active, reactive and apparent powers and power factor $\cos \varphi$ ) of the motor without the load (the disk) and in the presence of the misalignment fault were measured (Table 5).

The values of active, reactive and apparent powers and power factor $\cos \varphi$ in the case of both of angular and parallel misalignment are high compared to those obtained from the unloaded motor as shown in Table 5. The reason for this increase is that the vibration level is higher when the shaft is misaligned. Electrical values are remarkably increased in the case of parallel misalignment shaft compared to angular misalignment. Therefore, it can be

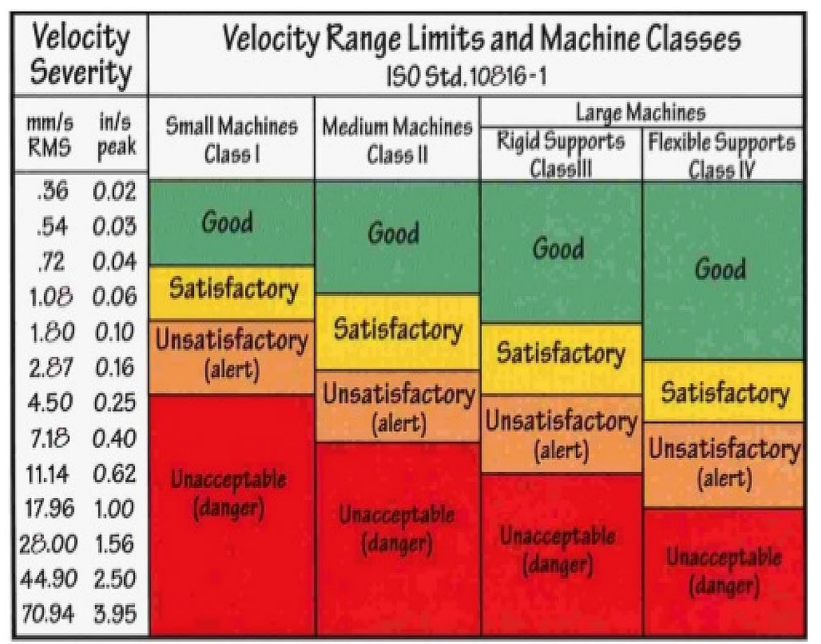

Fig. 5 ISO 10816-1 standards chart

concluded that the increase in vibration levels cause an unusual consumption of energy.

\section{Discussion}

As seen in the Fig. 6, parallel misalignment (case 3 ) is more severe because its RMS values are higher than those recorded in case of angular misalignment (case 2). Also, it has been noticed that measuring position P2 (bearing 1) shows the highest values compared to the other positions. This is due to the fact that the bearing 1 is close to the misalignment in comparison with the bearing 2 . Also, the bearings are more sensitive in the case of a misalignment
Table 3 RMS values in the defective case (angular misalignment)

Table 4 RMS values in the defective case (parallel misalignment)

\begin{tabular}{|c|c|c|c|c|c|c|}
\hline \multirow{3}{*}{$\begin{array}{l}\text { Measuring posi- } \\
\text { tion }\end{array}$} & \multicolumn{6}{|c|}{$\mathrm{RMS}(\mathrm{mm} / \mathrm{s})$} \\
\hline & \multicolumn{3}{|c|}{ Radial direction } & \multicolumn{3}{|c|}{ Axial direction } \\
\hline & $C_{A} 1$ & $C_{A} 2$ & $C_{A} 3$ & $C_{A} 1$ & $C_{A} 2$ & $C_{A} 3$ \\
\hline P1 & 0.103 & 1.395 & 1.861 & 0.189 & 1.321 & 1.796 \\
\hline P2 & 0.256 & 1.487 & 1.905 & 0.233 & 1.411 & 1.854 \\
\hline P3 & 0.098 & 1.214 & 1.714 & 0.097 & 1.193 & 1.582 \\
\hline
\end{tabular}

\begin{tabular}{|c|c|c|c|c|c|c|}
\hline \multirow{3}{*}{$\begin{array}{l}\text { Measuring posi- } \\
\text { tion }\end{array}$} & \multicolumn{6}{|c|}{$\mathrm{RMS}(\mathrm{mm} / \mathrm{s})$} \\
\hline & \multicolumn{3}{|c|}{ Radial direction } & \multicolumn{3}{|c|}{ Axial direction } \\
\hline & $C_{p} 1$ & $C_{p} 2$ & $C_{p} 3$ & $C_{p} 1$ & $C_{p} 2$ & $C_{p} 3$ \\
\hline P1 & 0.918 & 1.623 & 1.956 & 0.855 & 1.567 & 1.856 \\
\hline P2 & 1.267 & 1.887 & 2.006 & 1.305 & 1.731 & 1.936 \\
\hline P3 & 0.679 & 1.333 & 1.854 & 0.973 & 1.456 & 1.821 \\
\hline
\end{tabular}


Table 5 Energy balance

\begin{tabular}{|c|c|c|c|c|c|c|c|}
\hline \multirow[t]{2}{*}{ Electrical characteristics } & \multirow[t]{2}{*}{ Aligned shaft } & \multicolumn{3}{|c|}{ Angular misalignment } & \multicolumn{3}{|c|}{ Parallel misalignment } \\
\hline & & $C_{A} 1$ & $C_{A} 2$ & $C_{A} 3$ & $C_{p} 1$ & $C_{p} 2$ & $C_{p} 3$ \\
\hline Active power & 300 & 318 & 320 & 321 & 605 & 609 & 612 \\
\hline Reactive power & 930 & 953 & 955 & 959 & 958 & 962 & 969 \\
\hline Apparent power & 1000 & 1004 & 1007 & 1158 & 1133 & 1138 & 1305 \\
\hline Power factor $(\cos \varphi)$ & 0.29 & 0.30 & 0.30 & 0.31 & 0.51 & 0.51 & 0.53 \\
\hline
\end{tabular}

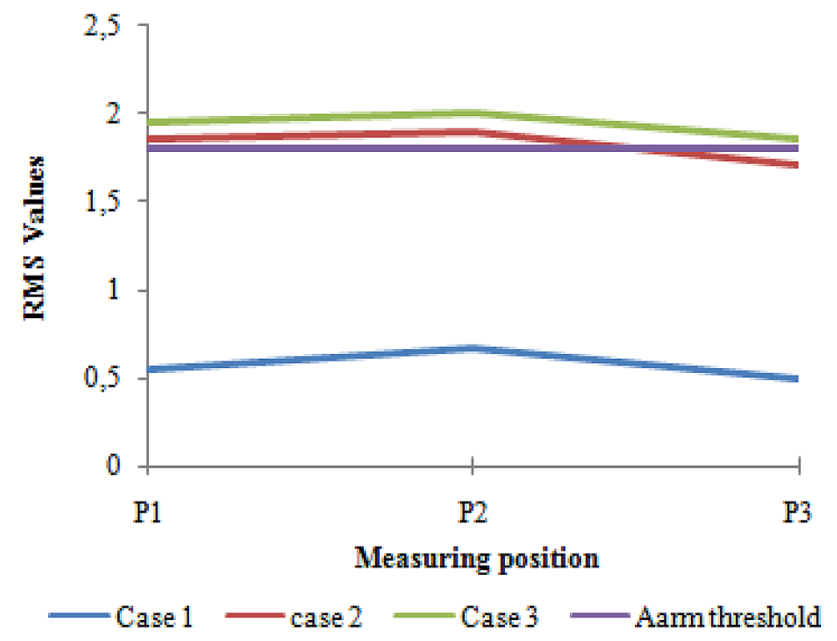

Fig. 6 Comparison of RMS values for studied cases

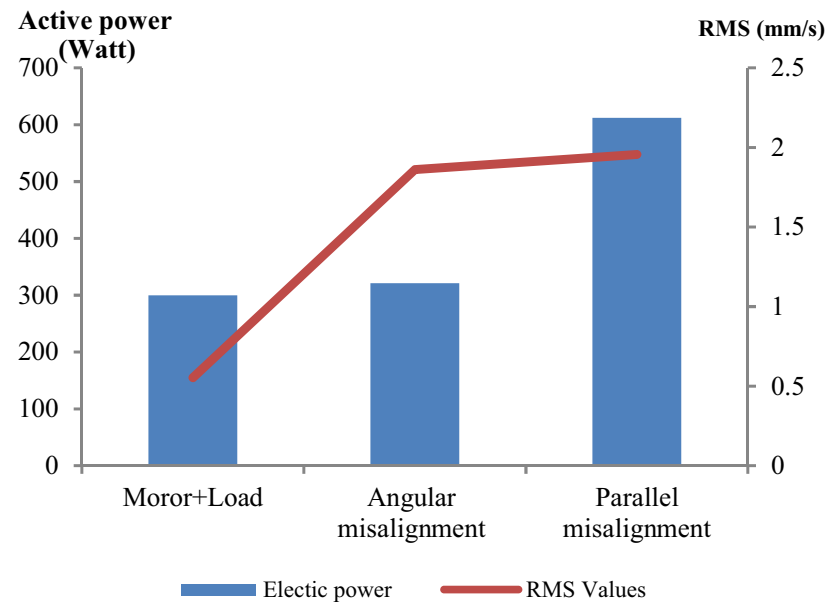

Fig. 7 Electrical power and RMS values in different cases

fault than the other organs of rotating machinery (for example: motor).

Now, to establish the relationship between vibration and energy consumption, Fig. 7 summarized the results obtained of the calculation of electrical power consumed by the motor in the different cases (Aligned shaft, parallel misalignment and angular misalignment).
Indeed, Fig. 7 shows that the vibration level and the energy consumption have changed with a similar tendency when the motor is more and more loaded and the shaft is misaligned. From all measurements, we observe that difference between the highest and lowest values of power consumption is more significant in the case of parallel misalignment.

In this research, both of parallel and angular shaft misalignment influenced the energy consumption because it was found that by switching from healthy to the defective state, the electrical power of the motor increases. This was an indication of the energy losses caused by misaligned shaft. It is in this perspective that we are proposing to use the RMS values to monitor both the vibratory level of the machine and its energy consumption.

These conclusions confirm the results of studies previously published by other authors; for example, E. Estupinan et al. [13], analyzed energy losses due to misalignment using current as an indicator of energy consumption. However, in our research, we interested to calculate electrical power and precisely the active power, which is the real power dissipated in the circuit. In addition, we show through this study that vibration analysis can be used effectively in predicting faults and monitoring energy consumption.

\section{Conclusion}

Vibration analysis is the best and sensitive techniques adopted in condition monitoring process. In this paper vibration analysis is used to study various misalignments fault conditions such as in parallel and in angular direction.

From the results of extensive experimental work on the designed test rig, it can be concluded that there is strong correlation between vibration levels and the power consumption. It has been shown that vibration measurements are effective indicator for the monitoring of energy consumption. Thus, the reliability of machines is a major responsible for the increase of energy consumption and vibration analysis technique may present an effective solution for the evaluation of energy consumption. 


\section{Compliance with ethical standards}

Conflict of interest The authors declare that they have no conflict of interest.

\section{References}

1. Nikolic AB (2015) Methodology for energy audits in power plants regarding analysis of electrical energy consumption. In: Conference paper, June 2015

2. Mobley RK (2002) An introduction to predictive maintenance, 2nd edn. Elsevier, Amsterdam, pp 99-113

3. Kumar S, Dhanabalan S, Narayanan CS (2019) Application of ANFIS and GRA for multi-objective optimization of optimal wireEDM parameters while machining Ti-6Al-4V alloy. SN Appl Sci 1:298

4. Duan L, Yao M, Wang J, Bai T, Zhang L (2016) Segmented infrared image analysis for rotating machinery fault diagnosis. Infrared Phys Technol 77:267-276

5. Khodaei M, Rafeeyan M, Ebrahimi S (2012) Energy loss calculation due to misalignment and unbalance in coupling-disk system. Mech J Modares 12:156-162

6. Piotrowski JA (2006) Shaft alignment handbook, 3rd edn. CRC Press, Boca Raton, pp 35-68

7. Chen $Q$, Yuan Q, Lei M, Wang M (2016) Shafting alignment computing method of new multibearing rotor system under specific installation requirement. Math Probl Eng 2016:1-12

8. Xu M, Marangoni R (1994) Vibration analysis of a motorflexible coupling-rotor system subject to misalignment and unbalance, part I: theoretical model and analysis. J Sound Vib 176(5):663-679
9. Xu M, Marangoni R (1994) Vibration analysis of a motor-flexible coupling-rotor system subject to misalignment and unbalance, part II: experimental validation. J Sound Vib 176(5):681-691

10. Sekhar AS, Prabhu BS (1995) Effects of coupling misalignment on vibrations of rotating machinery. J Sound Vib 185(4):655-671

11. Elkhatib A (2007) Energy consumption and machinery vibrations. In: 14th international congress on sound and vibration, 9-12 July 2007

12. Moghe SM, Mahure AN (2017) Study of vibration response of coupling under misalignment condition-a review. Int J Res Advent Technol 5(4):50-53

13. HA Gaberson (1996) Rotating machinery energy loss due to misalignment. In: IECEC 96, proceedings of the 31st intersociety energy conversion engineering conference, vol 3, pp 1809-1812

14. Estupinan E, Espinoza D, Fuentes A (2008) Energy losses caused by misalignment in rotating machinery: a theoretical, experimental and industrial approach. Int J COMADEM 11(2):12-18

15. Abouelanouar B, Elamrani M, Elkihel B, Delaunois F, Manssouri I (2017) A comparative experimental study of different methods in detection and monitoring bearing defects. Int J Adv Sci Tech Res 1(7):409-423

16. Corne B, Knockaert J, Desmet J (2017) Misalignment and unbalance fault severity estimation using stator current measurements. In 2017 IEEE 11th international symposium on diagnostics for electrical machines, power electronics and drives, $\mathrm{pp}$ 247-253

17. Dewell DL, Mitchell LD (1984) Detection of a misaligned disk coupling using spectrum analysis. J Vib Acoust 106(1):9

Publisher's Note Springer Nature remains neutral with regard to jurisdictional claims in published maps and institutional affiliations. 